\title{
Specialization in Surgery: Why It Is Important
}

\section{Mallika Tewari ${ }^{1}$}

Published online: 19 February 2019

(C) Association of Surgeons of India 2019

We all have heard the proverb: "practice makes perfect". This phrase originated in the mid-1500s in the American English language that was adopted from a Latin phrase "Uses promptos facit." The literary origin is in the work titled "Diary and Autobiography of John Adams" which was penned by Gregory Titelman. The saying has changed in the way it was used earlier from "Use makes perfect" to "practice makes perfect."

It is a glaring fact supported by data that high-volume hospitals and high-volume surgeons contribute to better patient outcomes and reduced mortality and hence, outcome and volume are closely related. High-volume hospitals offer larger array of state-of-art facilities, best equipments and instruments, wide range of specialists, better intensive care and interventionists, multidisciplinary teamwork, and other resources all of which may not be available at smaller hospitals. In addition, they are more likely to have highly experienced and high-volume surgeons on their panel. Hence, high-volume hospitals are better equipped to deliver complex perioperative care after high-risk surgeries. On the other hand, personal surgical skills and experience of the surgeon also significantly determines the outcome of a surgical intervention and its longterm results. For example, outcome of certain cancer operations like lung resection depends more on the perioperative care as more patients are likely to die of cardiac events, pneumonia, or respiratory complications rather than from hemorrhage or leak. Outcomes following procedures such as carotid endarterectomy and breast cancer, however, depends more on surgeons' skill and experience, as most patients do not require intensive postoperative management. In a widely quoted study by Birkmeyer et al. [1], surgeon-volume had a major impact on hospital-volume and the proportion varied according to the procedure: it was $100 \%$ for aortic-valve replacement, $54 \%$ for

Mallika Tewari

drmtewari@gmail.com

1 Chief, HPB \& GI Division, Department of Surgical Oncology, Institute of Medical Sciences, Banaras Hindu University, Varanasi, U.P. 221005, India pancreatic resection, $49 \%$ for coronary-artery bypass grafting, $46 \%$ for esophagectomy, $39 \%$ for cystectomy, and $24 \%$ for lung resection. Other studies have reported similar results in reference to thyroid and parathyroid resections and total knee arthoplasty. It has been observed that high-volume surgeons migrating to low-volume hospital still deliver good outcome. The "best" results follow high-volume surgeons.

Literature is replete with instances wherein meticulous surgery and attention to details has resulted in considerably better patient outcomes. A good example is introduction of Total Mesorectal Excision (TME) in rectal cancer surgery by Heald and Enker. Kapiteijn E, et al. [2] analyzed the data of two trials and reported that the local recurrence rate decreased from $16 \%$ in the older CRAB trial to $9 \%$ in the Dutch TME trial and the type of operation, conventional surgery (CRAB trial: in which conventional surgery was performed without quality control) versus TME (Dutch TME trial) was an independent predictor of local recurrence $(P=0.002)$; there was a higher survival rate in the Dutch TME trial. Similarly, less than optimal D2 resection in gastric cancer, low-volume surgeons and centers resulted in negative results of the Dutch and Medical Research Council trials in 1990s, the impact of which is still felt to-date wherein most centers in the West are skeptical of D2 gastric resections. Billroth, famous for his work on gastric cancer, had an $82 \%$ recurrence rate for breast cancer compared to Halsted, who reported only a $6 \%$ recurrence rate. Trained surgeons have consistently reported higher lymph node count following resections for colorectal and gastric cancer. Lymph node count, though it is dependent on several patient and tumor-related factors other than the surgeon, remains an important prognostic factor and adequate number of lymph nodes harvested (e.g., minimum 12 for colorectal and 15 for gastric cancer) prevents stage migration and hence under treatment of the patient. In their study, Morgan et al. [3], found that in hospitals with cancer program approval, $45.5 \%$ of the patients had 15 or more lymph nodes removed in gastric cancer surgeries versus $34.8 \%$ without cancer program approval. The above examples indicate that interest in a specific field results in improvement of knowledge and skills and patient outcomes. 
A look in to our own country, northern parts of India, wherein gallbladder cancer is common; it is not uncommon for patients to present with recurrence and metastatic disease following laparoscopic cholecystectomy elsewhere with apparently no operative and histopathology records. Similar is the fate of women undergoing suboptimal surgeries for breast cancer and cervix and ovarian malignancy. The seemingly evident conclusion from these observations is that surgeons should constantly assess their skill and ability of performing complex surgical procedures viz-a-viz the available hospital infrastructure and refer patients who need such procedures to centers of excellence.

Undeniably, a surgeon remains a prognostic factor and this is good news as this parameter is under our control. Efforts should be made to develop interest in a field and to constantly improve upon keeping abreast with the latest developments and innovations in the field of one's interest. A well-trained and well-supervised trainee will eventually make a good skilled surgeon with the ability to judiciously use the resources available and work in a multidisciplinary teamwork in compliance with international guidelines delivering optimal results. Interestingly, a meta-analysis also concluded that surgeons without fellowship training converted more laparoscopic operations to open surgery than those with fellowship training (risk ratio (RR) $1.04,95 \%$ c.i. 1.03 to 1.05 ) [4]. The fact that cannot be more emphasized is that the best opportunity to favorably influence the quality of any surgeon is a solid training period. Halsted credited with starting and Billroth were firm believers of a formal surgical residency-training program. A study by Are et al. analyzed data on the training paradigms from 174 countries and found significant global variations in the training paradigms of surgical oncologists calling a need for a global curriculum. In another recent study, Are et al. [5] reported an inverse relationship between the level of research activity and cancer-specific mortality. The USA was the most prolific country with 222,300 publications followed by Japan and Germany.

Hence, it is the need of the hour for young surgical trainees to sharpen their focus, select, develop, and innovate in their field of interest, and also contribute to research and publish.

\section{Compliance with Ethical Standards}

Conflict of Interest The author declares that there is no conflict of interest.

Publisher's Note Springer Nature remains neutral with regard to jurisdictional claims in published maps and institutional affiliations.

\section{References}

1. Birkmeyer JD, Siewers AE, Finlayson EV, Stukel TA, Lucas FL, Batista I, Welch HG, Wennberg DE (2002) Hospital volume and surgical mortality in the United States. N Engl J Med 346(15): 1128-1137

2. Kapiteijn E, Putter H, van de Velde CJ, Cooperative investigators of the Dutch ColoRectal Cancer Group (2002) Impact of the introduction and training of total mesorectal excision on recurrence and survival in rectal cancer in The Netherlands. Br J Surg 89(09):1142-1149

3. Morgan JW, Ji L, Friedman G, Senthil M, Dyke C, Lum SS (2015) The role of the cancer center when using lymph node count as a quality measure for gastric cancer surgery. JAMA Surg 150(1):37-43

4. Johnston MJ, Singh P, Pucher PH, Fitzgerald JE, Aggarwal R, Arora S, Darzi A (2015) Systematic review with meta-analysis of the impact of surgical fellowship training on patient outcomes. Br J Surg 102(10):1156-1166

5. Are C, Caniglia A, Malik M, Smith L, Cummings C, Lecoq C, Berman R, Audisio R, Wyld L (2018) Global variations in the level of cancerrelated research activity and correlation to cancer-specific mortality: proposal for a global curriculum. Eur J Surg Oncol 44(1):43-52 